\title{
Quantification of Ionic Transport within Thermally-Activated Batteries using Electron Probe Micro-Analysis
}

\author{
Thomas Humplik ${ }^{1}$, Emily K. Stirrup ${ }^{1}$, Anne M. Grillet ${ }^{1}{ }^{*}$, Richard P. Grant ${ }^{1}$, Ashley N. Allen ${ }^{1}$, \\ Daniel E. Wesolowski ${ }^{1}$, and Christine C. Roberts ${ }^{1}$ \\ 1. Sandia National Laboratories, PO Box 5800, Albuquerque, NM 87185
}

\begin{abstract}
The transient transport of electrolytes in thermally-activated batteries is studied using electron probe micro-analysis (EPMA), demonstrating the robust capability of EPMA as a useful tool for studying and quantifying mass transport within porous materials, particularly in difficult environments where classical flow measurements are challenging. By tracking the mobility of bromine and potassium ions from the electrolyte stored within the separator into the lithium silicon anode and iron disulfide cathode, we are able to quantify the transport mechanisms and physical properties of the electrodes including permeability and tortuosity. Due to the micron to submicron scale porous structure of the initially dry anode, a fast capillary pressure driven flow is observed into the anode from which we are able to set a lower bound on the permeability of 10-1 mDarcy. The transport into the cathode is diffusion-limited because the cathode originally contained some electrolyte before activation. Using a transient one-dimensional diffusion model, we estimate the tortuosity of the cathode electrode to be $2.8 \pm 0.8$.
\end{abstract}

\section{Keywords}

thermal battery, electron probe micro-analysis, electrolyte, mass transport, tortuosity, diffusion, permeability

*Corresponding Author: Anne M. Grillet - tel: (505) 844-7453; f: (505) 844-6620; amgrill@ sandia.gov 


\section{Introduction}

Molten salt (or thermally-activated) batteries utilize high melting temperature inorganic salts as the electrolyte [1]. Unlike conventional primary batteries, these batteries have a negligible self-discharge prior to activation due to the electrolyte's low ionic conductivity at room temperature; this property can lead to a shelf life in excess of 20 years [1]. Additionally, the $1-2$ order of magnitude higher ionic conductivity of the molten state compared to electrolytes used in standard lithium ion batteries (e.g., EC:DMC/LiPF 6 ) makes thermal batteries ideal for high voltage and high current applications [2-4]. Though molten salt batteries operate at elevated temperatures, the fundamentals of transport (both electronic and ionic) are not considerably different from those seen in typical 'room-temperature' batteries. After activation during which the temperature of the battery is raised in excess of the electrolyte melting temperature - power is provided when lithium discharges from the active material (lithiumsilicon) within the porous anode, transports through the molten salt electrolyte, and reacts with the active material (iron disulfide) within the porous cathode to complete the electrochemical reaction [5-7]. Like most batteries, the power performance is limited by the transport (i.e., the diffusion coefficient and permeability) of electrons within the solid-phase active materials and mobile ions through the liquid-phase electrolyte [8-10]. While experimental techniques for investigating ionic transport within batteries exist (such as impedance spectroscopy [11-13] or, more recently, neutron depth profiling [10]), it is often difficult to deduce the transport mechanisms within the individual electrodes. Recent work by Lu and Harris [14] used time-offlight secondary ion mass spectrometry (TOF-SIMS) to examine transport of lithium ions through the solid electrolyte interphase (SEI) which conformally coats the active material in lithium ion batteries. Surface sputtering allowed depth profiles to be measured through the 
thickness of the SEI ( 20nm). While TOF-SIMS can't measure quantitative chemical concentrations, it can accurately measure isotope ratios. By tracking the displacement of ${ }^{7} \mathrm{Li}$ with its isotope ${ }^{6} \mathrm{Li}$, the lithium transport was found to occur by a combination of diffusion in the porous outer portion of the SEI and a knock-off mechanism within the denser inner portion $[14][15]$.

Understanding and quantifying the ionic transport mechanisms throughout the electrodes is crucial to understand to understand battery operation. The components can be fabricated with different pore sizes, porosities, and methodologies (e.g., pressing with or without the electrolyte/binder): changing these properties can significantly effect not only the transport behavior, but also the battery performance. Therefore, novel experimental techniques are required to constrain the analysis to individual electrodes and thus improve the understanding of electrolyte mobility and ionic transport within these porous composites.

In this work, we report on a new method to measure electrolyte transport using an electron probe micro-analyzer (EPMA) to quantify the mobility of the ionic species during operation. EMPA combines the high spatial resolution of electron microscopy with the precise elemental detection $(0.1 \mathrm{wt} \%)$ of wavelength dispersive spectroscopy to create two-dimensional maps of the ionic concentration. We observed two different mechanisms of transport: a fast capillary-pressure driven flow of electrolyte into the anode, and a diffusion-limited flow into the tortuous cathode. From the analysis, we were able to set a lower limit on the electrolyte permeability into the anode and estimated a tortuosity of the cathode. While this work is a first step in the experimental quantification of mass transport within thermal batteries, this work demonstrates the robust capability of utilizing EPMA as tool to probe transport through micro- to macro-scale porous materials. 


\section{Experimental}

Single cells were constructed based on a typical molten salt battery configuration composed of an iron disulfide $\left(\mathrm{FeS}_{2}\right)$ cathode, a magnesium oxide $(\mathrm{MgO})$ and electrolyte separator, and a lithium silicon ( $\mathrm{LiSi})$ anode $[2,5,7,14]$. Two different electrolytes were used in this study: a eutectic lithium chloride-potassium chloride (45:55 wt\%, respectively) mixture with a corresponding melting point of $352^{\circ} \mathrm{C}$; and a lithium chloride-lithium bromide-potassium bromide (12:36.5:54.5 wt \%, respectively) mixture with a melting point of $325^{\circ} \mathrm{C}$. Each of the layers was individually pressed into a thin disk using standard methods described by Guidotti \& Reinhart [1]. The separator was composed of 70wt\% LiCl:LiBr:KBr electrolyte and 30wt\% $\mathrm{MgO}$. In the cathode, the $\mathrm{FeS}_{2}$ was mixed with the $\mathrm{LiCl}: \mathrm{KCl} / \mathrm{MgO}$ binder $(65 \mathrm{wt} \% / 35 \mathrm{wt} \%$, accounting for $25 \mathrm{wt} \%$ of the cathode) to improve the pressing properties as the active material alone does not form a cohesive pellet. The LiSi anode was pressed to a residual porosity of $15 \mathrm{vol} \%$ but without any embedded electrolyte.

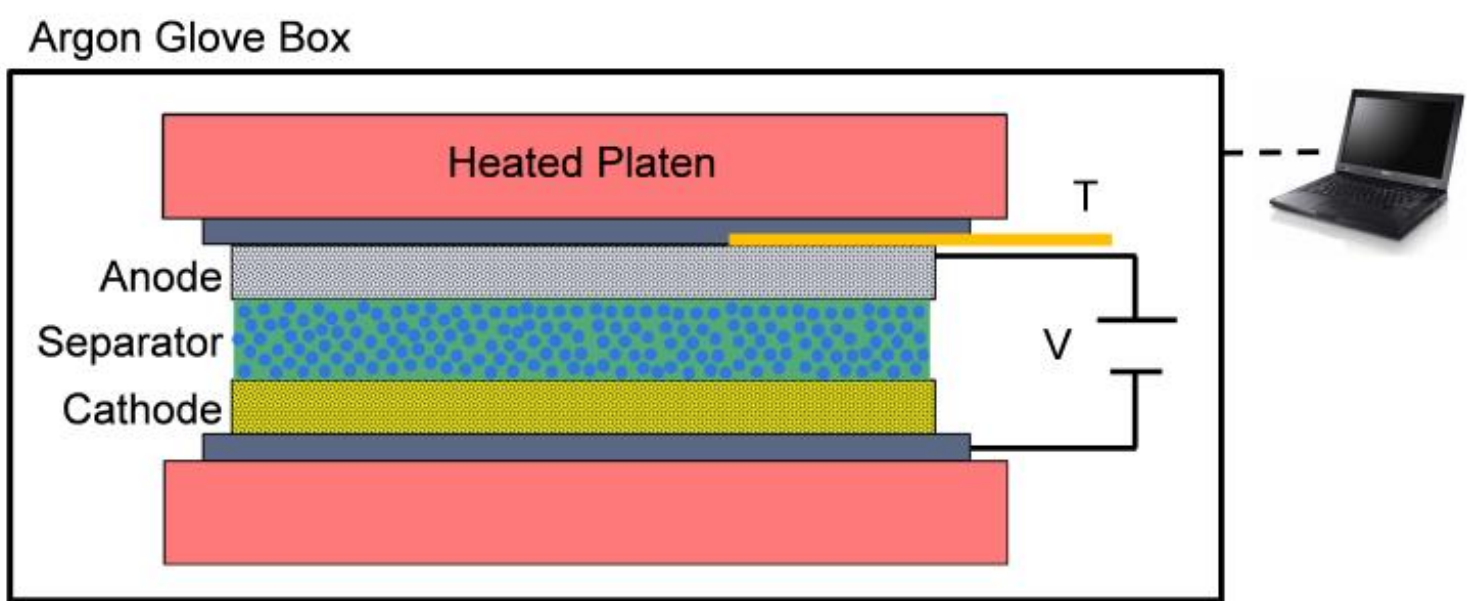

Figure 1: Schematic of the experimental setup used to test the molten salt cells [14]. Each cell was composed of an anode, a separator, and a cathode which were sandwiched between 2 stainless steel collector plates. The heated platens compressed the cells to a stress of $12 \mathrm{psi}$, which mimicked the conditions of typical battery activation. The temperature and open circuit voltage were recorded to 
determine the activated times. After a set time, the cells were removed and quenched to room temperature to freeze the electrolyte and stop transport.

Six 1.25 " diameter single battery cells were tested at open circuit conditions between heated platens inside of a dry, argon-filled glove box (Figure 1). Cells were fabricated by placing the anode, separator, and cathode between stainless steel shim stock. The cells were placed between the heated platens held at a constant temperature of $500^{\circ} \mathrm{C}$ for a controlled amount of time to allow for electrolyte migration. A uniaxial compressive stress of 12 psi was applied to hold the cell together and ensure good electrical contact between layers [6,14]. As reported elsewhere, the separator compresses by $\sim 20 \%$ on melting of the electrolyte [6] while no appreciable change in thickness was measured for the other two layers. The cell open circuit voltages (OCV) were measured by monitoring the voltage between the steel sheets using a DAQ system (USB-6251, National Instruments). Temperature was also monitored using a thermocouple attached to the top of the stainless shim. After a set duration, the cells were removed from the heated platens and quickly quenched by setting each cell on a room-temperature metal block to decrease the temperature below the electrolyte melting temperature. The cells took between 10 and 40 seconds to drop below the solidification temperature of the electrolyte which should halt ionic transport within the electrolyte phase.

After the cells had cooled, they were sectioned for electron microscopy. The quenched single cells were first coated in polystyrene and then potted in epoxy for cross-sectioning. The polystyrene protects the hygroscopic salts from potential water contamination due to the condensation curing reaction of the epoxy. The samples were polished using a water-free solution in a dry room to avoid water absorption [15]. Imaging and elemental mapping were performed using a JEOL JXA-8530F HyperProbe electron probe micro-analyzer with a $20 \mathrm{kV}$ 
beam and a $20 \mathrm{nA}$ current. The resulting electron beam spot size was $100 \mathrm{~nm}$ in diameter. For creation of the $2 \mathrm{D}$ cross section maps, the images were generated with a single pass using a 10 millisecond per point dwell time across $256 \mu \mathrm{m}$ by $256 \mu \mathrm{m}$ with measurements every $1 \mu \mathrm{m}^{2}$. While measurements were performed on 7 elemental constituents, bromine and potassium were of primary interest to study the transport of the electrolyte. A large lithium-fluoride crystal was used for bromine detection, and a high x-ray intensity pentaerythritol crystal was used for potassium detection. The excited x-rays from each pixel were collected with a proportional x-ray counter and were normalized against a known standard to generate a weight percent signal for each element. Furthermore, a ZAF ( $\mathbf{Z}$, the atomic number correction, $\mathbf{A}$ the absorption correction, $\mathbf{F}$ the fluorescence correction) correction was applied to the sample to improve the accuracy of the analysis. The measured chemical weight fractions should be accurate to $0.1 \mathrm{wt} \%$. It should be highlighted that a significant effort was made to minimize the exposure of the cells to any moisture in the air. The samples were kept within dry conditions, and transferred into the EPMA within a custom-made environmental chamber that interfaced with the loading chamber.

\section{Results and Discussion}

For all tests, the bromine-laden electrolyte started within the separator and was tracked into the cathode and anode to determine the mass transport properties. Prior to image analysis, the activated time for each cell was determined. The active time was quantified by averaging the time the cell was above $325^{\circ} \mathrm{C}$ (the temperature at which the bromine-based electrolyte melted), above $352^{\circ} \mathrm{C}$ (the temperature at which the $\mathrm{LiCl}: \mathrm{KCl}$ electrolyte melted), or at an $\mathrm{OCV}$ above $1.8 \mathrm{~V}$. The standard deviation of the averaged active time was also included to better quantify the 
uncertainty for the transport analysis. Figure 2 shows an example of the data collected from the 151 second cell while it was held between the heated platens (the data for all cells is provided in the supporting information). For all cells, both the temperature and OCV were above their respective thresholds for approximately the same amount of time. The compiled data for the three activation time measures for all cells is shown in Table 1 with the cell name representing the average value.

Table 1. Compiled activated time and average experimental temperature (when the electrolyte was above $1.8 \mathrm{~V}, 352^{\circ} \mathrm{C}$, and $325^{\circ} \mathrm{C}$ ) from data collected during the heating experiments. Note that the naming scheme of the cells was the average of all three values. The average temperatures from the experiments were used in the temperature-diffusion relations in Janz et al. to estimate the diffusion coefficient [17]. The calculated diffusion at these temperatures coefficient ranged from $8 \times 10^{-10} \mathrm{~m}^{2} \mathrm{~s}^{-1}$ (when $\mathrm{T}=392{ }^{\circ} \mathrm{C}$ ) to $1.6 \times 10^{-9} \mathrm{~m}^{2} \mathrm{~s}^{-1}$ (when $\mathrm{T}=470^{\circ} \mathrm{C}$ ).

\begin{tabular}{|c|c|c|c|c|}
\hline Cell name & $\mathrm{V}>1.8 \mathrm{~V}$ & $\mathrm{~T}>352{ }^{\circ} \mathrm{C}$ & $\mathrm{T}>325^{\circ} \mathrm{C}$ & $\mathrm{T}_{\text {AVE }}\left({ }^{\circ} \mathrm{C}\right)$ \\
\hline $1 \mathrm{sec}$ & 4 & 0 & 0 & -- \\
\hline $37 \mathrm{sec}$ & 30 & 33 & 47 & 392 \\
\hline $38 \mathrm{sec}$ & 27 & 39 & 46 & 406 \\
\hline $87 \mathrm{sec}$ & 74 & 84 & 101 & 425 \\
\hline $151 \mathrm{sec}$ & 133 & 151 & 167 & 460 \\
\hline $260 \mathrm{sec}$ & 238 & 263 & 287 & 470 \\
\hline
\end{tabular}




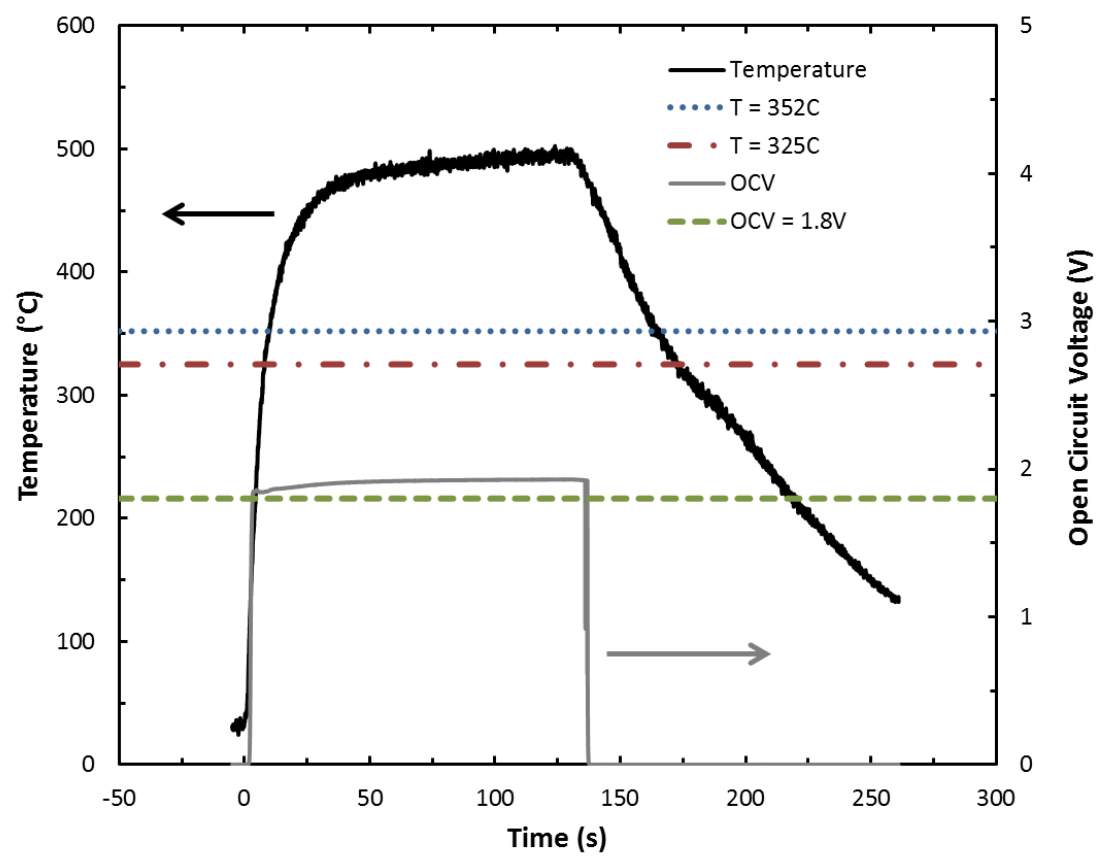

Figure 2: Experimental open circuit voltage (OCV) and temperature data for the 151 second cell. The activated times were averaged from the amount of time that the cell was at an OCV $>1.8 \mathrm{~V}$ (red dashed line) at a temperature greater than $352^{\circ} \mathrm{C}$ (blue dotted line - which corresponds to the melting temperature of the LiCl: $\mathrm{KCl}$ electrolyte), or a temperature greater than $325^{\circ} \mathrm{C}$ (green dash-dotted line which corresponds to the melting temperature of the $\mathrm{LiCl}: \mathrm{LiBr}: \mathrm{KBr}$ electrolyte). More data is provided in the supporting information (Figures S1 and S2), while the tabulated experimental results are shown in Table 1.

After initial imagining with standard scanning electron microscopy, each cell was surveyed with the EPMA to generate elemental concentration maps for both bromine and potassium. The elemental maps were generated for each respective component (e.g., cathode, separator and anode), and subsequently were stitched together to form a complete single cell (the combined images can be seen in Figure 3). Image processing of these combined maps was performed using a custom MatLab code. A threshold value of $2 \mathrm{wt} \%$ of either potassium or bromine was applied to each pixel to ensure the image analysis was limited to the electrolyte/binder within the cell (i.e., the solid constituents and void space were removed from the analysis and appear as a neutral gray in Figure 3). In presenting results, a ratio of bromine and potassium is used to 
account for variations in electrolyte concentration in each measurement volume. The

normalization (referred to henceforth as the normalized bromine concentration) is defined as:

$$
\overline{B r}=\frac{B r(w t \%)}{B r(w t \%)+K(w t \%)}
$$

Initially, the normalized bromine concentration (in molar fraction/molar fraction) was $\approx 0.66$ in the separator and 0 in both the anode and cathode. The final equilibrium concentration was $\approx 0.55$ across the entire cell. The results of this analysis and compiled mapping are shown in Figure 3.

a)

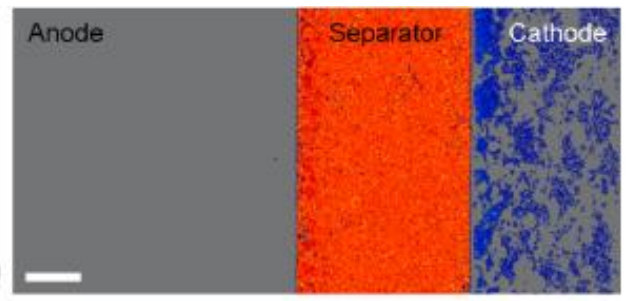

b)

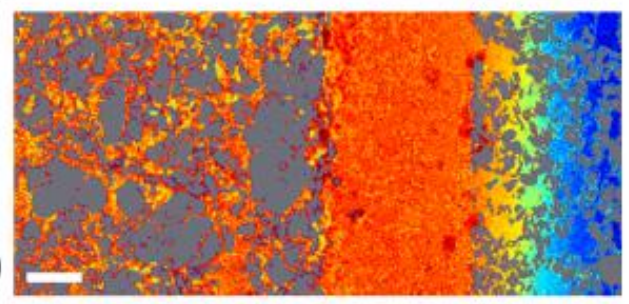

c)

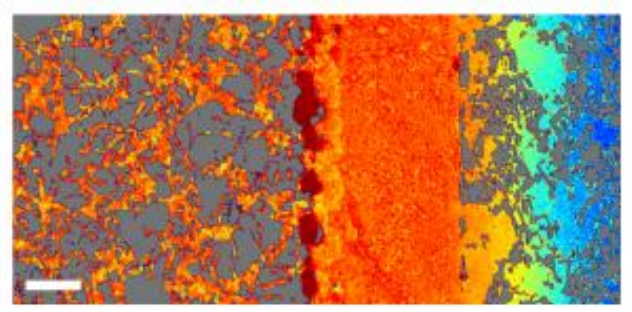

d)

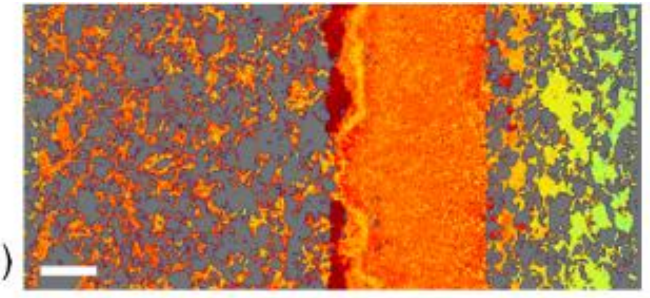

e)

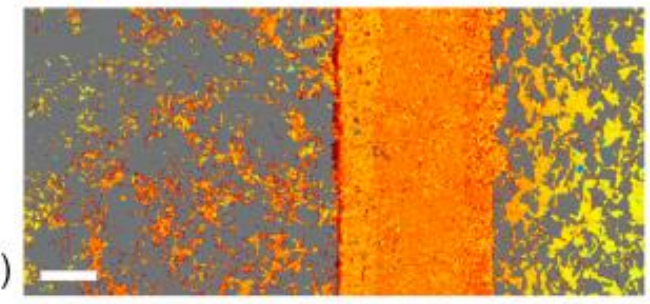

f)

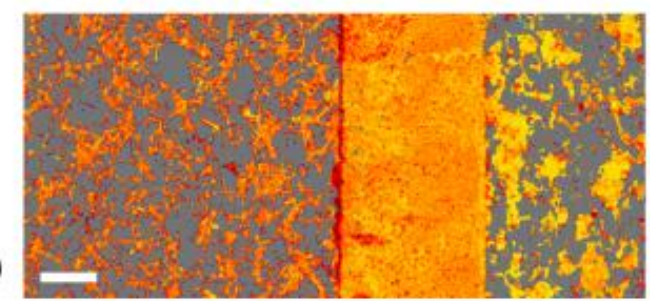

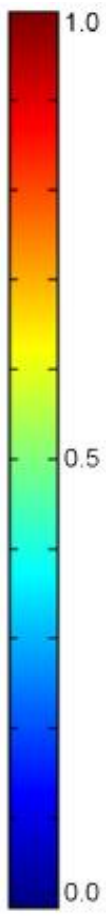

Figure 3: Compiled maps of the normalized bromine concentration for the cells activated for various times. a) $1 \pm 1$ seconds b) $37 \pm 9$ seconds c) $38 \pm 11$ seconds d) $87 \pm 14$ seconds e) $151 \pm 19$ seconds f) $260 \pm 21$ seconds. Areas in grey are either void space or the solid ( $\mathrm{LiSi}$ or $\left.\mathrm{FeS}_{2}\right)$ matrix. Once the electrolyte in the separator fully melted (between 1 and 37 seconds), the separator became thinner and the electrolyte quickly filled the initially dry LiSi anode structure. This fast transport is evidence of a large capillary pressure that forced the electrolyte to wick into the anode structure. The bromine transport into 
the cathode was slower and appeared to be diffusion-limited. An equilibrium normalized bromine concentration across all cells was reached between 151 and 260 seconds. The scale bar for all images is $100 \mu \mathrm{m}$.

Initially, the bromine-based electrolyte was located only within the separator (refer the SEM in Figure 3A). For an active time of 1 second (Figure 3A), it appeared that the electrolyte had not melted during the experiment and there was no electrolyte exchange with either the cathode or the anode. Though this cell reached OCV, the EPMA imaging covered a cross-section of $\approx 1 \mathrm{~mm}^{2}$ out of a diameter of $31.7 \mathrm{~mm}$ in size, so we hypothesize that we examined an area where the electrolyte had not yet melted ${ }^{1}$. A substantial change occurred between an active time

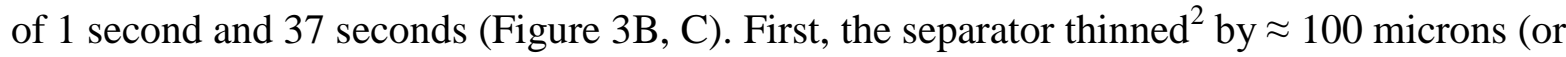
$20 \%$ of the initial thickness), indicating that the melted electrolyte both filled a majority of the void space (e.g., air pockets) within the separator and also transported into the cathode and anode [6]. The initially dry anode structure was wetted with the bromine-based electrolyte. In contrast, the transport into the cathode was markedly slower. However, since the cathode was originally pre-mixed with the $\mathrm{LiCl}: \mathrm{KCl}$ electrolyte, this behavior was expected as the bromine-based electrolyte had to diffuse across the electrode. Over the next $\approx 220$ seconds (Figures 3D-F), the bromine-based electrolyte continues to diffuse from the separator into the cathode, and, correspondingly, the normalized bromine concentration decreased within the separator.

Equilibrium in the normalized bromine concentration across the electrodes and the separator was reached between an active time of 151 seconds and 260 seconds.

It is also clear from the images that the structure inside the electrode is very heterogeneous which is typical of composite electrode of many types of batteries. The resulting

\footnotetext{
${ }^{1}$ Because the OCV was reached, the electrolyte did melt somewhere within the cell. We believe this behavior could be indicative of a non-uniform temperature within the cell.

${ }^{2}$ It has been well-reported that the magnesium oxide binder rearranges during this activation process and thins until it reaches a solid-like structure $[1,6,14]$.
} 


\footnotetext{
${ }^{3}$ The normalized bromine concentration was averaged along the horizontal direction (or the y-axis of the images) of the images in Figure 3 to obtain a statistical average (and corresponding standard error of the mean).

${ }^{4}$ This analysis assumes that the permeability of the separator is larger than the anode, and therefore not limiting the flow into the anode.

${ }^{5}$ Time-varying changes in the porosity and porous flow were considered to be second-order terms and were neglected in this initial analysis.
} 


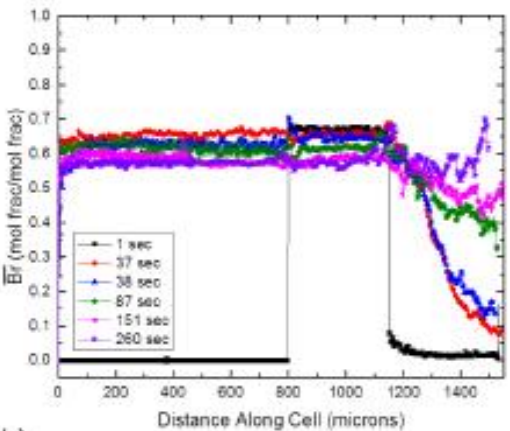

b)

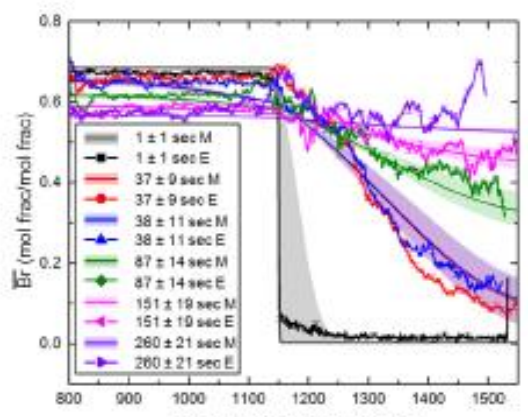

c)

Figure 4: a) Scanning electron microscopy image of the cross-section of one of the cells (87 seconds) used for the transport experiments. The bright contrast in the anode structure is the electrolyte while the darker areas are lithium-silicon. In the cathode, the brighter contrast areas correspond to the iron disulfide matrix while the darker areas are the electrolyte. b) Normalized bromine concentration $-\mathrm{Br} /(\mathrm{Br}+\mathrm{K})-$ across the cells for the various active times. Note the abrupt rise in bromine concentration in the anode in comparison with the slower increase in concentration for the cathode. c) The electrolyte transport between separator and cathode was modeled to estimate the tortuosity of the cathode. The image shows the experimental ( $\mathrm{E}$ - line/symbol) and modeled ( $\mathrm{M}$ - lines with shaded regions) concentration profiles for the separator and cathode of the cells studied in this work. Note that the origin of the abscissa is the interface between the anode and separator (e.g., $x=800 \mu \mathrm{m})$. For the modeling results, the average time is the solid line, while the standard deviation in the time corresponds to the respective shaded region for each average. The diffusivity of the electrolyte in the model was set to $1 \times 10^{-9} \mathrm{~m}^{2} \mathrm{~s}^{-1}$ [17] and a tortuosity of $2.8 \pm 0.8$ was found to match the experimental concentration profiles.

While the temperature-diffusion relationships for these types of salts are only experimentally verified for temperatures in excess of $600^{\circ} \mathrm{C}$, we extrapolated these relations to lower temperatures to estimate the diffusivity of bromine and potassium at the temperatures observed during the experiments [17]. For each experiment, the diffusivity at the average cell 
temperature noted in Table 1 was determined; values ranged from $8 \times 10^{-10} \mathrm{~m}^{2} \mathrm{~s}^{-1}$ to $1.6 \times 10^{-9} \mathrm{~m}^{2} \mathrm{~s}^{-1}$. Because the diffusion coefficient and the tortuosity are coupled (i.e., the higher the diffusivity, the larger the tortuosity), we solved for the relation between the diffusivity and tortuosity that best matched the concentration profiles. The best solution is shown in Figure 4C. The experimental data is the line/symbol, while the modeling results for the average activation time is shown with a solid line. The shaded regions around the modeling results show the range of results corresponding to standard deviation for the activation time. The best fit for the experimental data was observed for a tortuosity of $2.8 \pm 0.8$ using an average value for diffusion coefficient of $1 \times 10^{-9} \mathrm{~m}^{2} \mathrm{~s}^{-1}$. Uncertainties in the diffusion coefficient impact the solution for the tortuosity. Clearly, if more information about either the microstructure or the diffusion coefficient was known, that would also reduce the uncertainty.

The results from these experiments demonstrate the high spatial and elemental resolution of EPMA as a tool to better understand the mechanisms of mass transport through porous materials, particularly for applications that operate in challenging (i.e., high temperature and corrosive) environments. While the results from these experiments alone do not provide a complete picture of the thermal battery transport properties due to challenges with temporal resolution limiting the extracted quantitative analysis, they offer a first step in understanding electrolyte mobility within thermally-activated batteries. Therefore, future experiments are planned to improve the characterization of these electrodes to better understand the transport properties of the molten electrolyte through the cathode, as well as the effects of pre-applied load, temperature, and porosity/tortuosity on the diffusion and flow of electrolyte.

\section{Conclusions}


The transport of electrolyte within a molten salt battery was tracked through the use of elemental mapping via Electron Probe Micro-Analysis. A large $(\approx 20$ - 40 psi) capillary pressure drove a fast wicking behavior of the electrolyte from the separator into the anode and wetted the electrode within the first $37 \pm 9$ seconds after thermal activation. The lower limit on the anode permeability was determined to be $10^{-1} \mathrm{mDarcy}$. The transport of the electrolyte from the separator into the cathode was slower due to the diffusion-limited transport into the previously electrolyte-filled cathode. The diffusion profiles were modeled using a time-varying onedimensional diffusion equation. Using a diffusion coefficient of $1 \times 10^{-9} \mathrm{~m}^{2} / \mathrm{s}$ for the bromine and potassium, the tortuosity was calculated to be $2.8 \pm 0.8$. An improved knowledge of either the electrode microstructure or the electrolyte diffusion coefficient could significantly decrease the standard deviation in calculated results. Nevertheless, these experiments demonstrated the capability of utilizing EPMA as a useful tool for studying transport through porous media, particularly in environments where more typical permeability measurements are challenging due to temperature or material compatibility.

\section{Acknowledgements}

The authors acknowledge Bonnie McKenzie (Sandia National Laboratories) for the initial SEM imaging of the electrodes. The authors would also like to graciously thank Scott Roberts (Sandia National Laboratories) and Kevin Long (Sandia National Laboratories) for helpful discussions throughout the work.

This work was funded through the Joint Munitions Program and Engineering Campaign C6. Sandia National Laboratories is a multi-program laboratory managed and operated by Sandia Corporation, a wholly owned subsidiary of Lockheed Martin Corporation, for the U.S. Department of Energy's National Nuclear Security Administration under contract DE-AC0494AL85000. SAND2015-8856 J 


\section{References}

[1] R. A. Guidotti, P. Masset, Thermally activated ("thermal") battery technology. Part I: An overview, J. Power Sources. 161 (2006) 1443-1449. doi:10.1016/j.jpowsour.2006.06.013.

[2] P. Masset, R. A. Guidotti, Thermal activated (thermal) battery technology. Part II. Molten salt electrolytes, J. Power Sources. 164 (2007) 397-414. doi:10.1016/j.jpowsour.2006.10.080.

[3] S.S. Zhang, T.R. Jow, K. Amine, G.L. Henriksen, LiPF $6 \pm$ EC \pm EMC electrolyte for Li-ion battery, J. Power Sources. 107 (2002) 18-23. doi:10.1016/S03787753(01)00968-5.

[4] A. Nyman, M. Behm, G. Lindbergh, Electrochemical characterisation and modelling of the mass transport phenomena in LiPF6-EC-EMC electrolyte, Electrochim. Acta. 53 (2008) 6356-6365. doi:10.1016/j.electacta.2008.04.023.

[5] P.J. Masset, R. A. Guidotti, Thermal activated ("thermal") battery technology. Part IIIa. $\mathrm{FeS}_{2}$ cathode materials, J. Power Sources. 177 (2008) 595-609. doi:10.1016/j.jpowsour.2007.11.017.

[6] R.A. Guidotti, F.W. Reinhardt, Characterization of MgO Powders for Use in Thermal Batteries, SAND Report 90-2104, Sandia National Laboratories, Sandia National Laboratories, 1996.

[7] R. A. Guidotti, P.J. Masset, Thermally activated ("thermal") battery technology. Part IV. Anode materials, J. Power Sources. 183 (2008) 388-398. doi:10.1016/j.jpowsour.2008.04.090.

[8] K. Persson, V. A. Sethuraman, L.J. Hardwick, Y. Hinuma, Y.S. Meng, A. Van Der Ven, et al., Lithium diffusion in graphitic carbon, J. Phys. Chem. Lett. 1 (2010) 11761180. doi: $10.1021 /$ jz100188d.

[9] B. Scrosati, Lithium Rocking Chair Batteries: An Old Concept?, J. Electrochem. Soc. 139 (1992) 2776. doi:10.1149/1.2068978.

[10] D.X. Liu, J. Wang, K. Pan, J. Qiu, M. Canova, L.R. Cao, et al., In situ quantification and visualization of lithium transport with neutrons, Angew. Chemie - Int. Ed. (2014) 9498-9502. doi:10.1002/anie.201404197.

[11] M.D. Levi, D. Aurbach, Diffusion coefficients of lithium ions during intercalation into graphite derived from the simultaneous measurements and modeling of electrochemical impedance and potentiostatic intermittent titration characteristics of thin graphite electrodes, J. Phys. Chem. B. 101 (1997) 4641-4647. doi:10.1021/jp9701911.

[12] P. Prosini, M. Lisi, D. Zane, M. Pasquali, Determination of the chemical diffusion coefficient of lithium in LiFePO4, Solid State Ionics. 148 (2002) 45-51. doi:10.1016/S0167-2738(02)00134-0.

[13] D. Aurbach, Review of selected electrode-solution interactions which determine the performance of Li and Li ion batteries, J. Power Sources. 89 (2000) 206-218. doi:10.1016/S0378-7753(00)00431-6.

[14] P. Lu, S. Harris, Lithium transport within the solid electrolyte interphase, Electrochem. Commun. 13 (2011) 1035-1037. doi:10.1016/j.elecom.2011.06.026.

[15] S. Shi, P. Lu, Z. Liu, Y. Qi, L.G. Hector, H. Li, S. Harris, Direct Calculation of Li-Ion Transport in the Solid Electrolye Interphase, J. Am. Chem. Soc. (2012) 15476-15487. doi:10.1021/ja305366r. 
Table 1. Compiled activated time (when the electrolyte was above $1.8 \mathrm{~V}, 352^{\circ} \mathrm{C}$, and $325^{\circ} \mathrm{C}$ ) and average experimental temperature from experiments. Note that the naming scheme of the cells was the average of all three values. The average temperatures from the experiments were used in the temperature-diffusion relations in Janz et al. to estimate the diffusion coefficient [17]. The calculated diffusion at these temperatures coefficient ranged from $8 \times 10^{-10} \mathrm{~m}^{2} \mathrm{~s}^{-1}$ (when $\mathrm{T}=392{ }^{\circ} \mathrm{C}$ ) to $1.6 \times 10^{-9} \mathrm{~m}^{2} \mathrm{~s}^{-1}$ (when $\mathrm{T}=470^{\circ} \mathrm{C}$ ).

\begin{tabular}{|c|c|c|c|c|}
\hline Cell name & $\mathrm{V}>1.8 \mathrm{~V}$ & $\mathrm{~T}>352{ }^{\circ} \mathrm{C}$ & $\mathrm{T}>325^{\circ} \mathrm{C}$ & $\mathrm{T}_{\text {AVE }}\left({ }^{\circ} \mathrm{C}\right)$ \\
\hline $1 \mathrm{sec}$ & 4 & 0 & 0 & -- \\
\hline $37 \mathrm{sec}$ & 30 & 33 & 47 & 392 \\
\hline $38 \mathrm{sec}$ & 27 & 39 & 46 & 406 \\
\hline $87 \mathrm{sec}$ & 74 & 84 & 101 & 425 \\
\hline $151 \mathrm{sec}$ & 133 & 151 & 167 & 460 \\
\hline $260 \mathrm{sec}$ & 238 & 263 & 287 & 470 \\
\hline
\end{tabular}




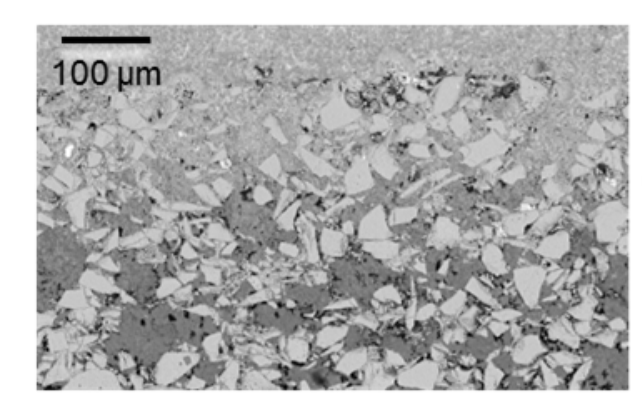

BSE SEM Image

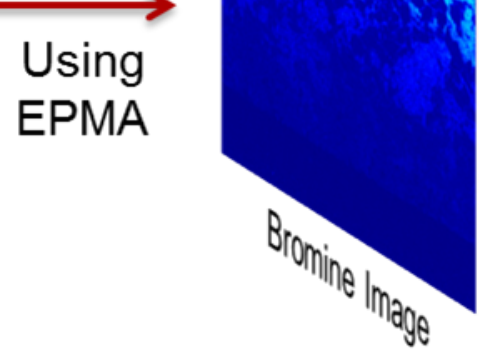

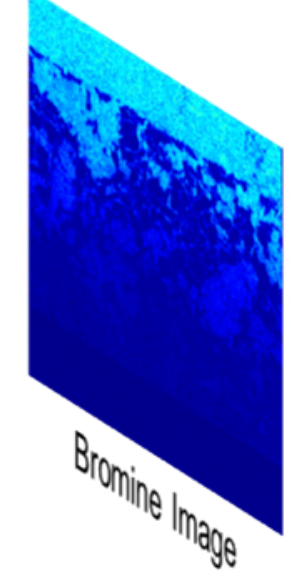

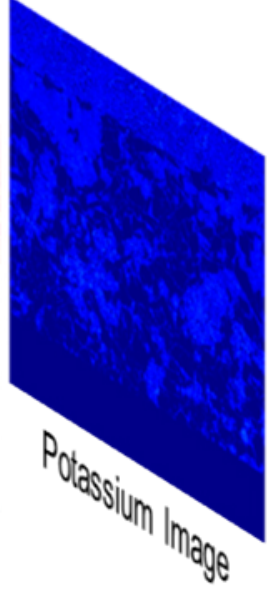

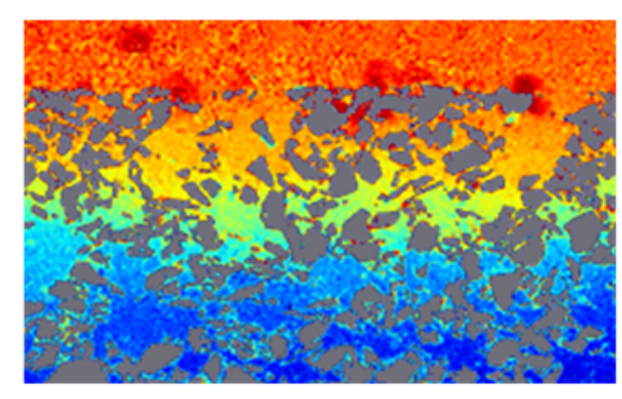

Normalized

Bromine Concentration
Image

Analysis 


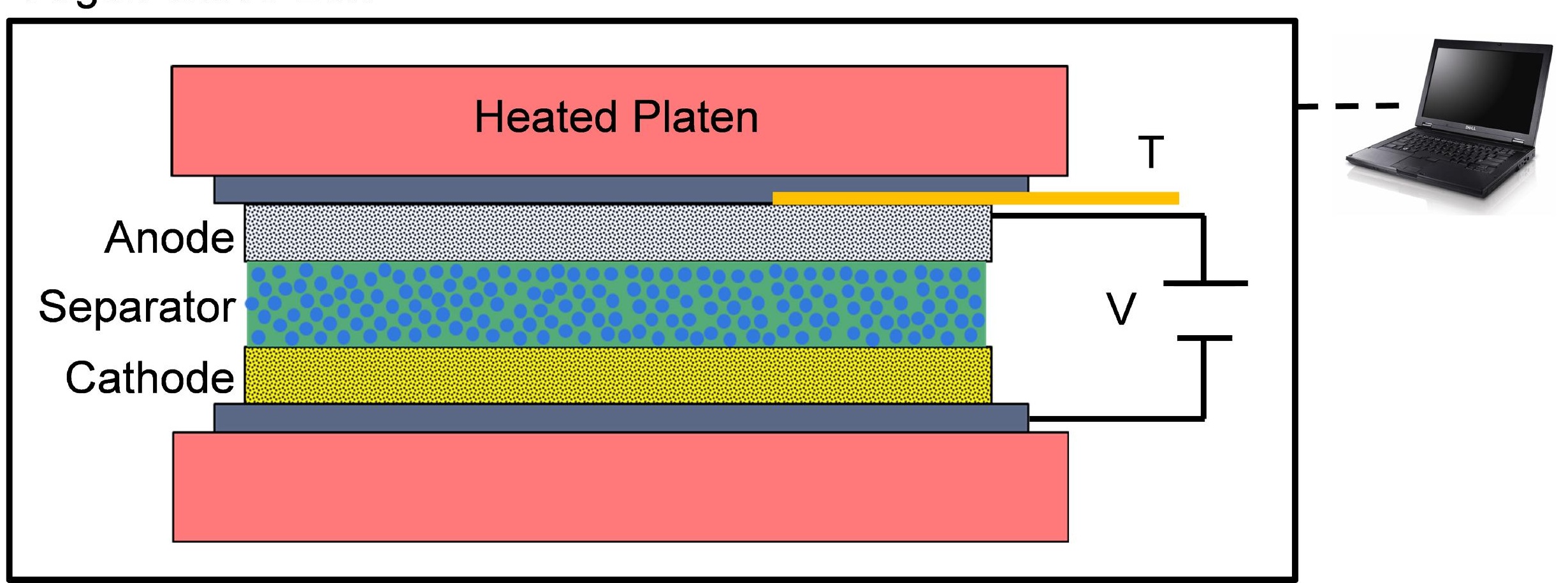




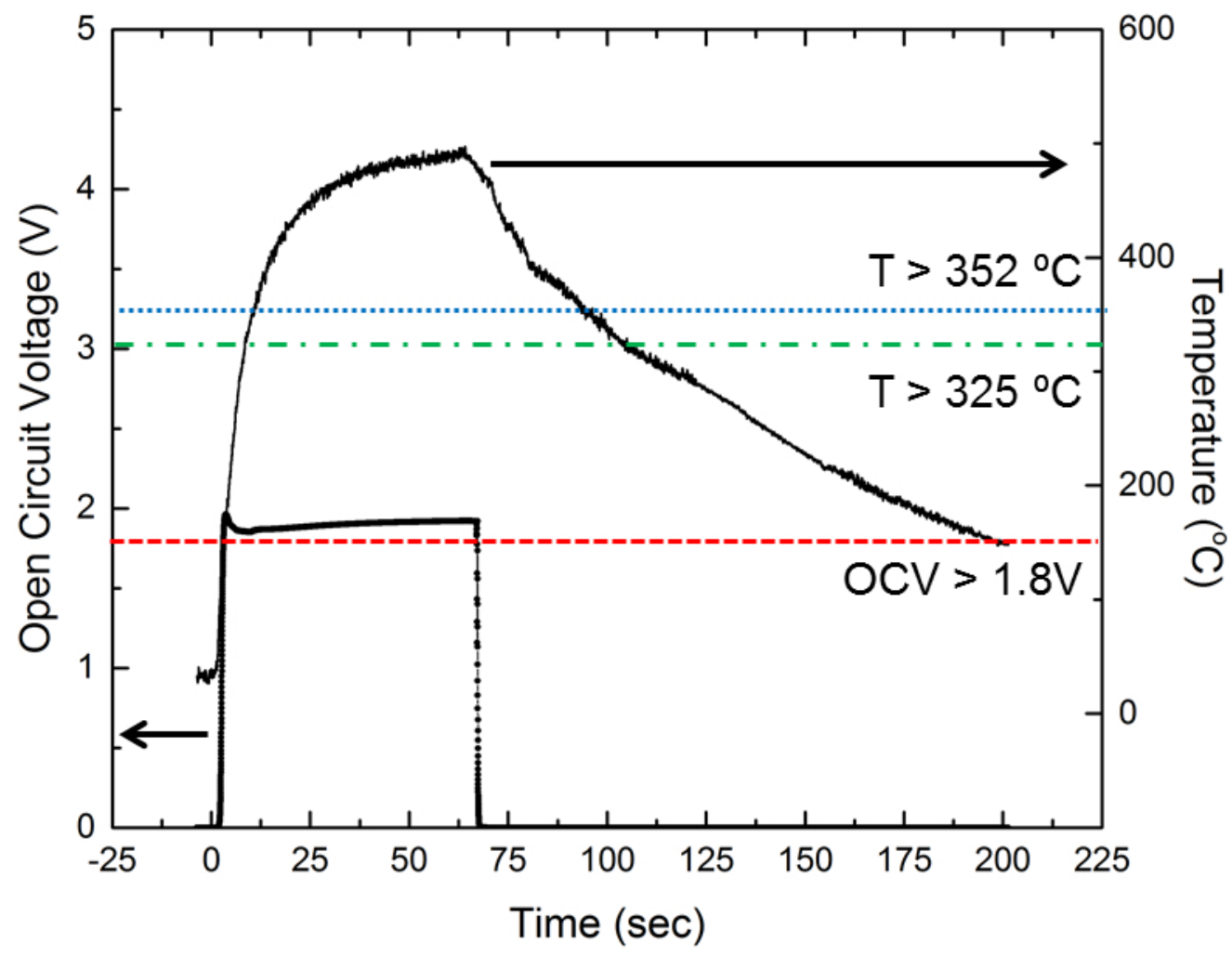




\section{Figukєеље}

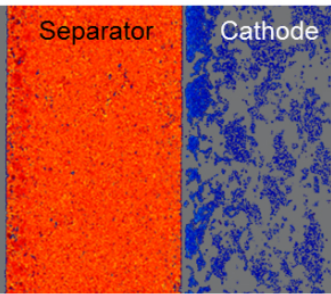

d) 1 -
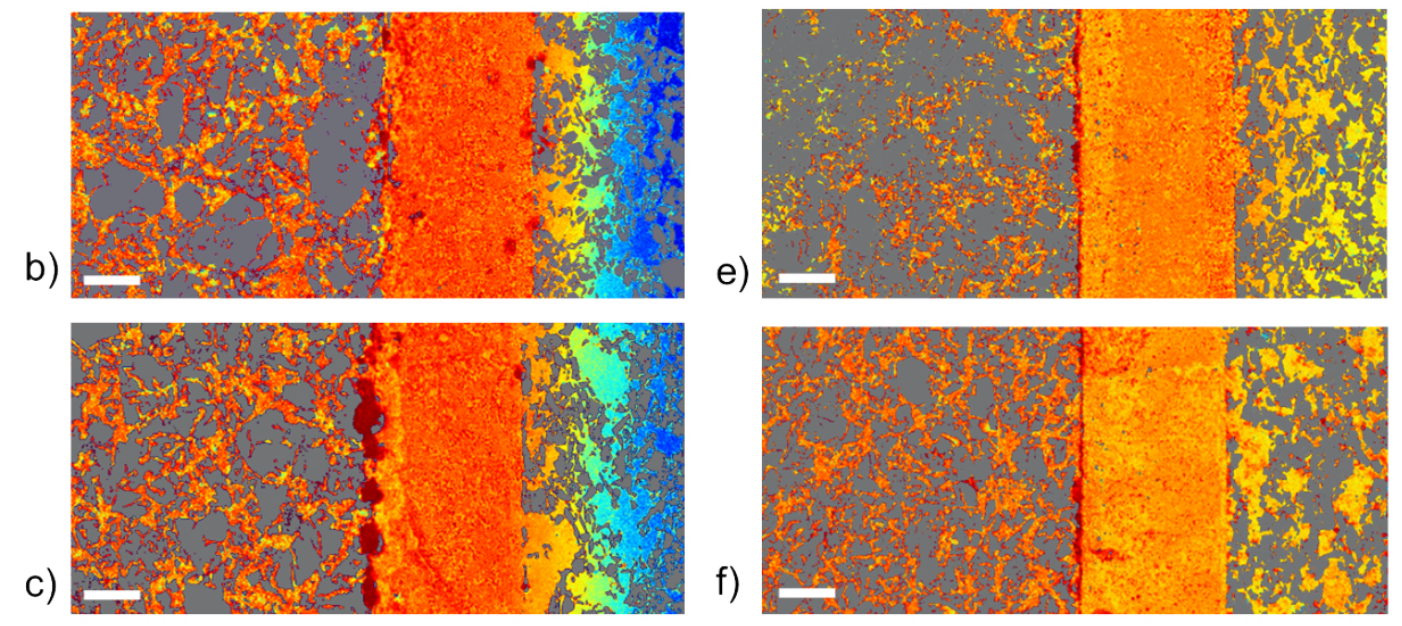


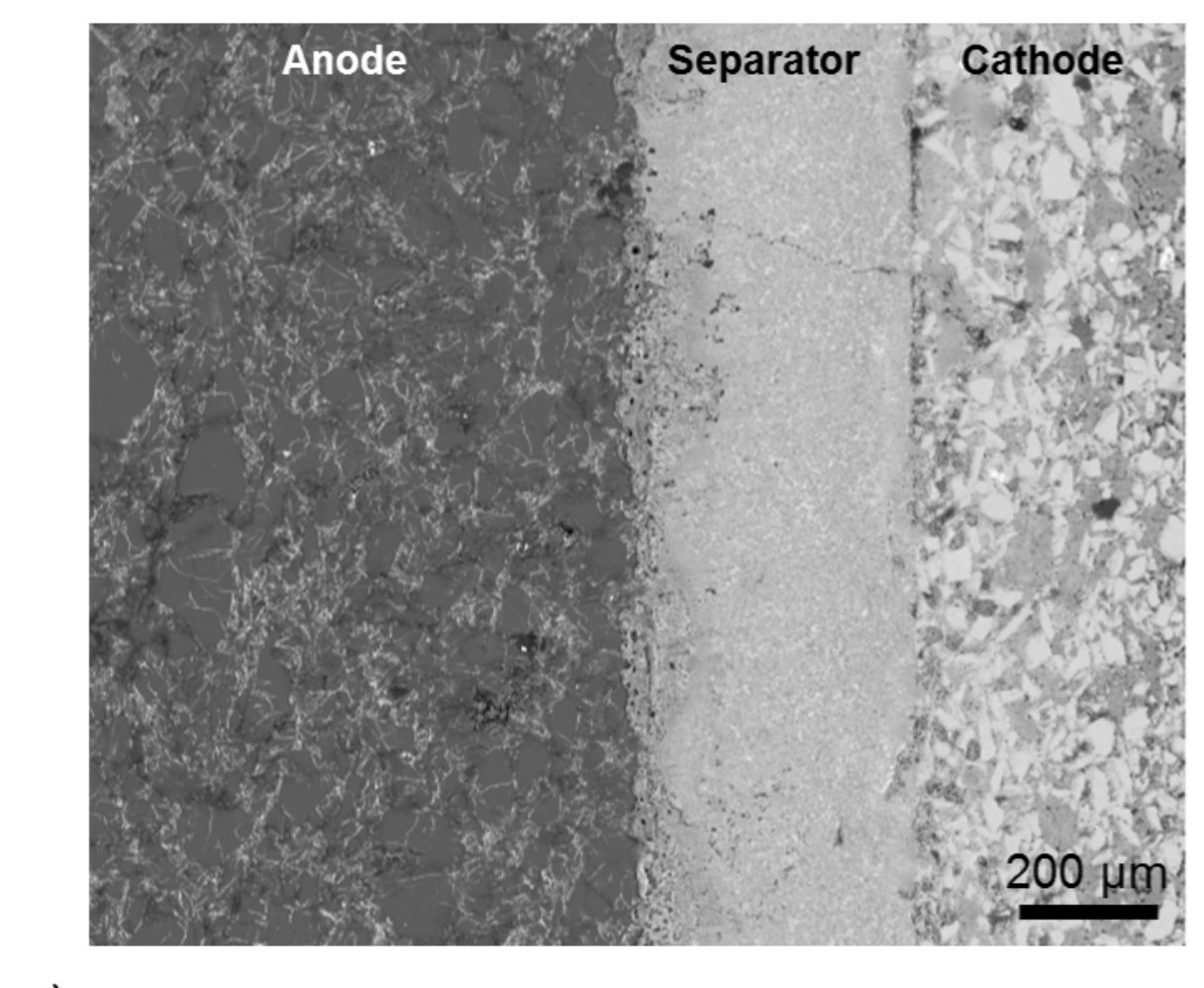

a)

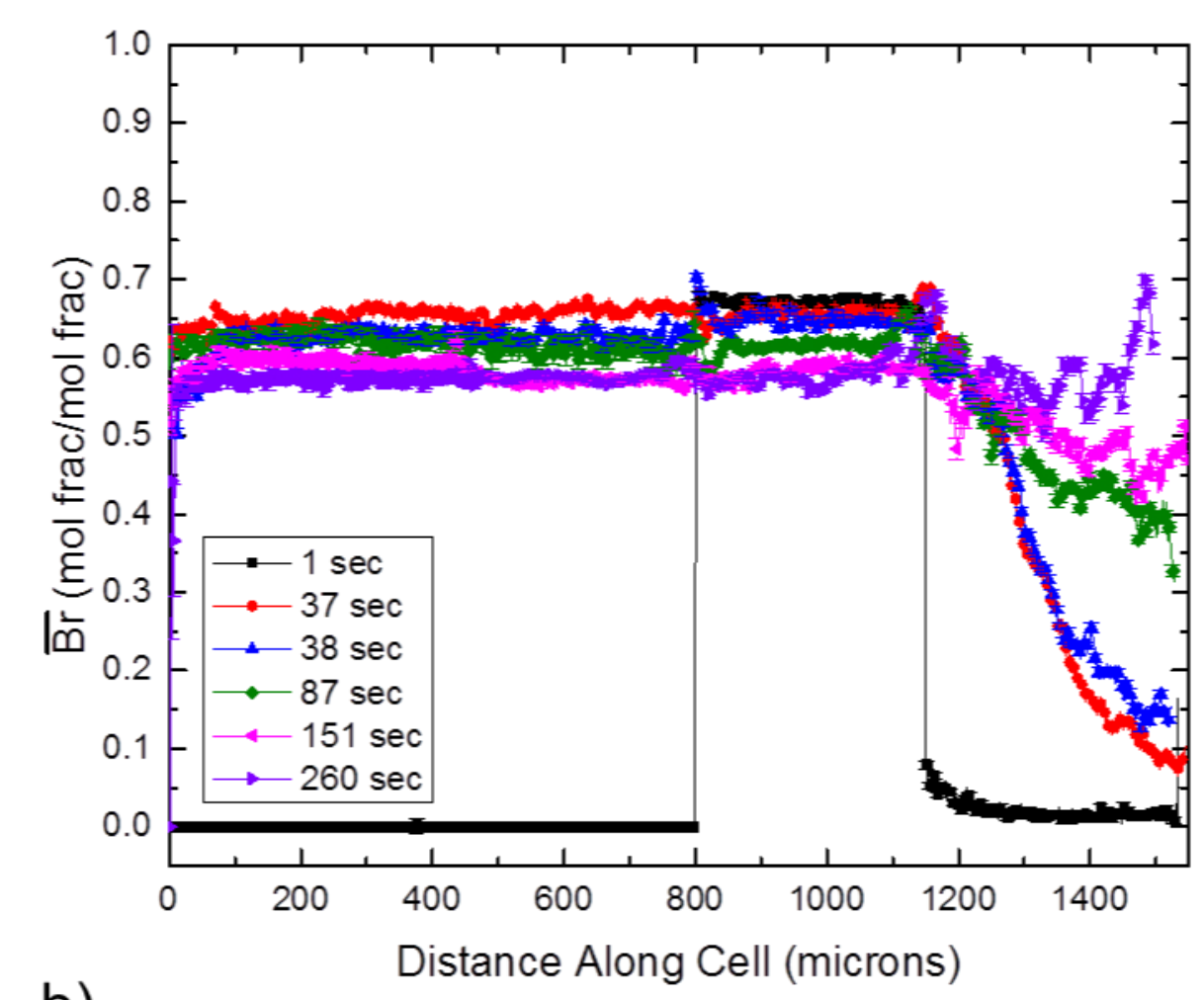

b)

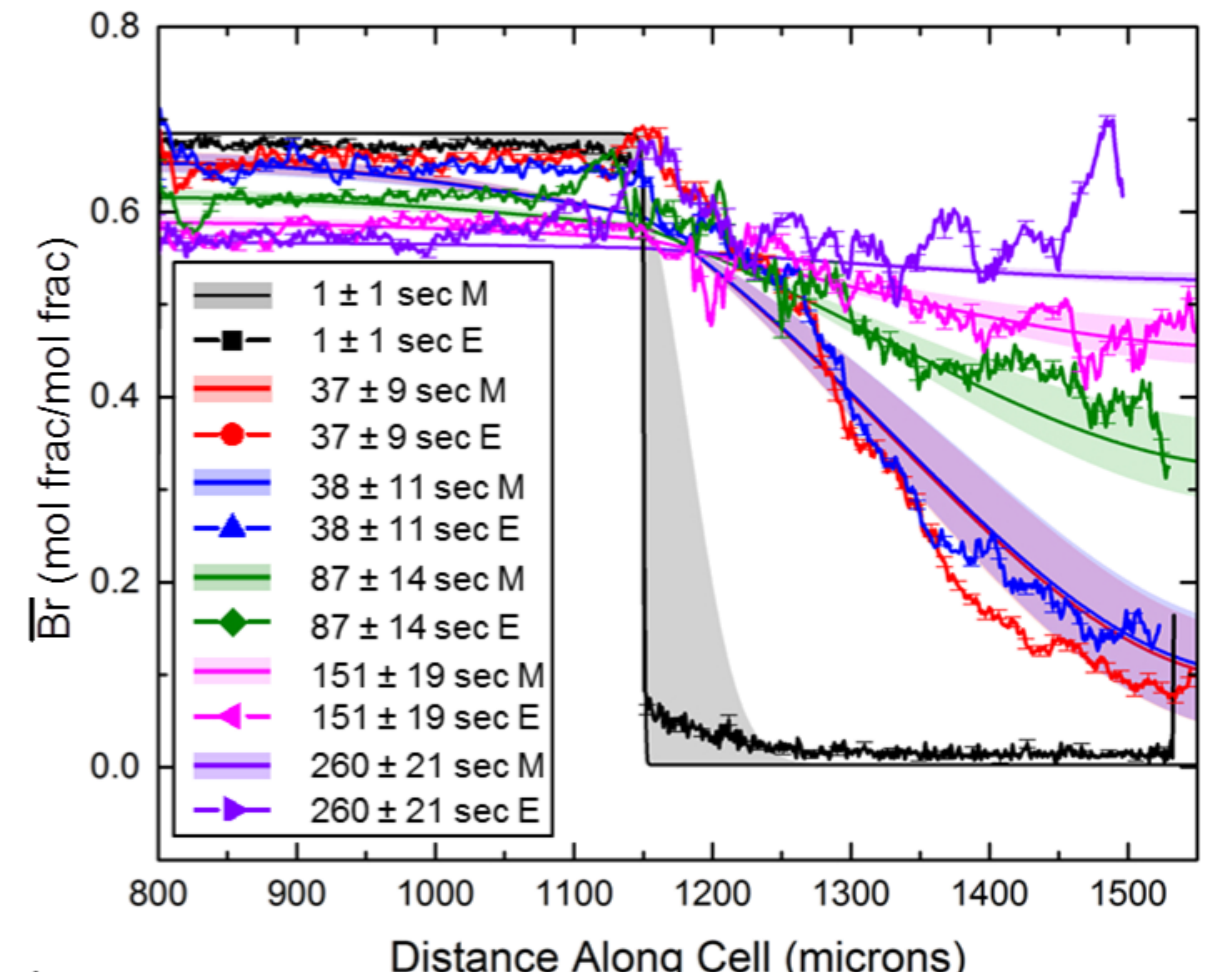

c) 


\section{Figure Captions}

Figure 1: Schematic of the experimental setup used to test the molten salt cells [14]. Each cell was composed of an anode, a separator, and a cathode which were sandwiched between 2 stainless steel collector plates. The heated platens compressed the cells to a stress of $12 \mathrm{psi}$, which mimicked the conditions of typical battery activation. The temperature and open circuit voltage were recorded to determine the activated times. After a set time, the cells were removed and quenched to room temperature to freeze the electrolyte and stop transport.

Figure 1: Experimental open circuit voltage (OCV) and temperature data for the 151 second cell. The activated times were averaged from the amount of time that the cell was at an OCV $>1.8 \mathrm{~V}$ (red dashed line) at a temperature greater than $352^{\circ} \mathrm{C}$ (blue dotted line - which corresponds to the melting temperature of the $\mathrm{LiCl}: \mathrm{KCl}$ electrolyte), or a temperature greater than $325^{\circ} \mathrm{C}$ (green dash-dotted line which corresponds to the melting temperature of the $\mathrm{LiCl}: \mathrm{LiBr}: \mathrm{KBr}$ electrolyte). More data is provided in the supporting information (Figures S1 and S2), while the tabulated experimental results are shown in Table 1.

Figure 2: Compiled maps of the normalized bromine concentration for the cells activated for various times. a) $1 \pm 1$ seconds b) $37 \pm 9$ seconds c) $38 \pm 11$ seconds d) $87 \pm 14$ seconds e) $151 \pm 19$ seconds f) $260 \pm 21$ seconds. Areas in grey are either void space or the solid ( $\mathrm{LiSi}$ or $\left.\mathrm{FeS}_{2}\right)$ matrix. Once the electrolyte in the separator fully melted (between 1 and 37 seconds), the separator became thinner and the electrolyte quickly filled the initially dry LiSi anode structure. This fast transport is evidence of a large capillary pressure that forced the electrolyte to wick into the anode structure. The bromine transport into the cathode was slower and appeared to be diffusion-limited. An equilibrium normalized bromine concentration across all cells was reached between 151 and 260 seconds. The scale bar for all images is $100 \mu \mathrm{m}$.

Figure 3: a) Scanning electron microscopy image of the cross-section of one of the cells (87 seconds) used for the transport experiments. The bright contrast in the anode structure is the electrolyte while the darker areas are lithium-silicon. In the cathode, the brighter contrast areas correspond to the iron disulfide matrix while the darker areas are the electrolyte. b) Normalized bromine concentration $-\mathrm{Br} /(\mathrm{Br}+\mathrm{K})-$ across the cells for the various active times. Note the abrupt rise in bromine concentration in the anode in comparison with the slower increase in concentration for the cathode. c) The electrolyte transport between separator and cathode was modeled to estimate the tortuosity of the cathode. The image shows the experimental ( $\mathrm{E}$ - line/symbol) and modeled ( $\mathrm{M}$ - lines with shaded regions) concentration profiles for the separator and cathode of the cells studied in this work. Note that the origin of the abscissa is the interface between the anode and separator (e.g., $\mathrm{x}=800 \mu \mathrm{m})$. For the modeling results, the average time is the solid line, while the standard deviation in the time corresponds to the respective shaded region for each average. The diffusivity of the electrolyte in the model was set to $1 \times 10^{-9} \mathrm{~m}^{2} \mathrm{~s}^{-1}$ [17] and a tortuosity of $2.8 \pm 0.8$ was found to match the experimental concentration profiles. 